\title{
Télescope
}

Revue d'analyse comparée en administration publique

\section{La gouvernance multiniveau dans les nations minoritaires : les cas du Québec-Canada et de la Catalogne-Espagne}

\section{Alexandre Couture Gagnon}

Volume 19, numéro 1, hiver 2013

La gouvernance multiniveau

URI : https://id.erudit.org/iderudit/1017156ar

DOI : https://doi.org/10.7202/1017156ar

Aller au sommaire du numéro

Éditeur(s)

L'Observatoire de l'administration publique

ISSN

1929-3348 (numérique)

Découvrir la revue

Citer cet article

Couture Gagnon, A. (2013). La gouvernance multiniveau dans les nations minoritaires : les cas du Québec-Canada et de la Catalogne-Espagne. Télescope, 19(1), 156-175. https://doi.org/10.7202/1017156ar

\section{Résumé de l'article}

Dans cet article, nous examinons la gouvernance multiniveau de deuxnations minoritaires (le Québec et la Catalogne) dans troischamps de politique, soit la langue, l'immigration et lesrelations internationales. Après l'examen de ces troischamps, il ressort qu'une telle gouvernance est absente de lapolitique linguistique au Québec-Canada, alors qu'elleatteint un paroxysme de formalisme pour ce qui est de la politiqued'immigration. Quant aux relations internationales, lagouvernance varie selon les dossiers. Du côté de laCatalogne-Espagne, la gouvernance multiniveau est appeléeà changer au chapitre de la politique linguistique, et elle estprésente en regard de la politique d'immigration,quoiqu'elle connaisse certaines difficultés en raison dela gestion budgétaire et de l'implication des éluspolitiques. Finalement, la politique de relations extérieuresest soumise à la volonté de Madrid. 


\title{
LA GOUVERNANCE MULTINIVEAU DANS LES NATIONS MINORITAIRES : LES CAS DU QUÉBEC-CANADA ET DE LA CATALOGNE-ESPAGNE
}

\author{
Par Alexandre Couture Gagnon 1, Professeure, École nationale d'administration \\ publique • alexandre.couture-gagnon@enap.ca
}

\begin{abstract}
RÉSUMÉ Dans cet article, nous examinons la gouvernance multiniveau de deux nations minoritaires (le Québec et la Catalogne) dans trois champs de politique, soit la langue, l'immigration et les relations internationales. Après l'examen de ces trois champs, il ressort qu'une telle gouvernance est absente de la politique linguistique au Québec-Canada, alors qu'elle atteint un paroxysme de formalisme pour ce qui est de la politique d'immigration. Quant aux relations internationales, la gouvernance varie selon les dossiers. Du côté de la CatalogneEspagne, la gouvernance multiniveau est appelée à changer au chapitre de la politique linguistique, et elle est présente en regard de la politique d'immigration, quoiqu'elle connaisse certaines difficultés en raison de la gestion budgétaire et de l'implication des élus politiques. Finalement, la politique de relations extérieures est soumise à la volonté de Madrid.
\end{abstract}

\begin{abstract}
In this article, I examine multi-level governance of two minority nations (Québec and Catalonia) in three policy areas; namely, language, immigration and foreign relations. From this analysis, it appears that multi-level governance is absent from linguistic policy in Québec-Canada while, on the other hand, immigration policy is highly formalized in terms of jurisdictional role. Regarding foreign policy, governance varies depending on the particular issue area. As concerns Catalonia-Spain, multi-level governance in the area of linguistic policy is bound to change. It is also present in the area of immigration policy, although it has encountered certain difficulties related to fiscal management and the involvement of elected representatives. Finally, foreign relations is a policy area that comes under the exclusive authority of Madrid.
\end{abstract}

Pour citer cet article : Couture Gagnon, A. (2013). « La gouvernance multiniveau dans les nations minoritaires : les cas du Québec-Canada et de la Catalogne-Espagne », Télescope, vol. 19, n 1, p. 156-175.

L e Québec et la Catalogne ${ }^{2}$ sont deux nations minoritaires du monde industrialisé qui portent une langue et une culture distinctes de celles du pays dans

\footnotetext{
L'auteure remercie les professeurs André Lecours, Tove Malloy, Leslie Pal et Stanley Winer ainsi que deux réviseurs anonymes pour leurs commentaires. Un merci tout spécial est adressé à l'European Centre for Minority Issues (ECMI, Flensburg, Allemagne) où I'auteure a effectué une partie de sa recherche. L'auteure remercie aussi les nombreux interviewés en vertu de l'approbation d'éthique numéro 12-0987 de I'Université Carleton. Toute erreur relève de la responsabilité de l'auteure. Cette recherche a bénéficié du financement de l'Association internationale des études québécoises (AIEQ), du Fonds québécois de la recherche sur la société et la culture (FQRSC), du Conseil de recherches en sciences humaines du Canada (CRSH), du Régime de bourses d'études supérieures de I'Ontario (BESO) et de l'Université Carleton.

2 Par Catalogne, nous désignons la communauté autonome d'Espagne nommée ainsi. Les Pays catalans comprennent d'autres régions de la France, de l'Espagne, de l'Italie et l'Andorre.
} 
lequel elles se trouvent. De surcroît, ces nations minoritaires sont soutenues par des entités politiques : le Québec est une province canadienne et la Catalogne, une communauté autonome espagnole. L'Espagne peut être qualifiée de quasifédération, tandis que le Canada est une fédération; à ce titre, la Catalogne perçoit ses revenus en moins grande proportion que le Québec. La Catalogne et le Québec revendiquent tous deux davantage de pouvoir dans les champs de politique publique qui définissent leur spécificité ou dans ceux qui les rapprochent d'un statut de quasi-État. Ainsi, les trois champs de politique sélectionnés pour cette étude sont la langue, l'immigration et les relations internationales, lesquels nous paraissent caractéristiques, voire emblématiques, des nations minoritaires. La langue et l'immigration sont susceptibles de garantir ou, à l'inverse, de mettre en danger l'avenir de la nation minoritaire. Si celle-ci ne préserve pas sa langue ou si ses immigrants ne l'apprennent pas, le futur de la nation minoritaire sera en péril. Quant aux relations internationales d'une nation minoritaire, elles lui permettent de se distinguer des autres entités non fédérales - c'est-à-dire des autres provinces au Canada et des autres communautés autonomes en Espagne - du pays. De plus, ces relations offrent une reconnaissance internationale, à l'image de celle à laquelle les véritables pays ont droit.

Dans l'ensemble des pays, et plus particulièrement au sein des fédérations, le partage des responsabilités est source de conflit entre les différents ordres de gouvernement. Chacun souhaite ne pas être responsable des compétences coûteuses ou peu susceptibles d'engager les citoyens. En contrepartie, chaque palier tente d'obtenir la responsabilité des compétences importantes aux yeux des citoyens; la loyauté de ces derniers est garante de réélection et de prestige en plus de faciliter la mise en œuvre de politiques ou de programmes auxquels le gouvernement en place tient profondément. Dans les pays comptant des nations minoritaires, l'enjeu du partage des responsabilités est encore plus grand, puisque certaines compétences revêtent un caractère vital pour la continuation de la nation minoritaire et deviennent ainsi encore plus précieuses aux yeux des deux ordres de gouvernement. C'est le cas des champs de politiques linguistiques, d'immigration et de relations internationales discutées ici.

Comment la gouvernance multiniveau se déploie-t-elle au Québec-Canada et en Catalogne-Espagne dans ces trois champs de politique ? Autrement dit, comment fonctionne la gouvernance d'un champ de politique lorsque deux ordres de gouvernement en sont (ou aimeraient en être) responsables ? Nous définissons la gouvernance multiniveau comme l'ensemble des processus par lesquels les deux paliers de gouvernement agencent leur politique respective dans un champ de politique donné. Ainsi, nous étudions la gouvernance séparément dans chacun des trois champs de politique étudiés. Pour chacun des trois champs de politique et pour chacun des cas (soit le Québec-Canada et la Catalogne-Espagne), nous déterminons s'il y a ou non gouvernance multiniveau entre les deux paliers gouvernementaux. Les résultats de l'analyse sont dichotomiques : soit il y a gouvernance multiniveau, soit celle-ci n'est pas présente. Si les deux paliers de gouvernement se consultent régulièrement en matière d'élaboration de politique et d'implantation de programme dans un champ de politique, nous considérons qu'il y a présence de 
gouvernance multiniveau. En revanche, si aucun document officiel ne démontre de collaboration et si les interlocuteurs interviewés affirment qu'il n'existe pratiquement aucun contact avec l'autre palier dans le champ de compétence étudié, nous tenons pour acquis qu'il n'y a pas de gouvernance multiniveau.

Notre texte s'inscrit dans une approche positive; il ne souhaite pas prescrire comment la gouvernance devrait être effectuée entre les ordres de gouvernement. Il vise uniquement à la décrire, sans porter de jugement normatif.

\section{- LA GOUVERNANCE MULTINIVEAU AU QUÉBEC-CANADA}

Au Québec-Canada, la gouvernance multiniveau diffère pour les trois champs de politique étudiés, soit la langue, l'immigration et les relations internationales. Ainsi, elle est inexistante pour les politiques linguistiques, puisque le Québec et le Canada conduisent leur politique de langue en vase clos et renvoient les conflits sporadiques au système judiciaire. À l'opposé, la gouvernance multiniveau atteint un très haut niveau dans le domaine de l'immigration. L'Accord Canada-Québec relatif à l'immigration et à l'admission temporaire des aubains de 1991 (ci-après, Accord Canada-Québec) stipule entre autres comment les deux gouvernements interagissent, quelle information ils doivent se transmettent et selon quelles modalités. Cette entente guide toutes les relations entre le Québec et le Canada au sujet de l'immigration. Entre les champs de politique linguistique et d'immigration se trouve celui des relations internationales. La gouvernance multiniveau y existe en partie (par exemple, pour les organisations internationales comme l'UNESCO), mais pas dans tous les dossiers (par exemple, pour le commerce international).

\section{La politique linguistique}

Dans le domaine de l'aménagement linguistique, la gouvernance multiniveau entre le Québec et le Canada n'existe quasiment plus depuis au moins les années 1990. Le Québec est seul responsable de sa politique linguistique dans ses champs de compétence, le Canada possédant une politique linguistique différente. En pleine Révolution tranquille, le rapport de la Commission royale d'enquête sur l'enseignement dans la province de Québec (Rapport Parent) recommanda l'intervention du gouvernement québécois " pour protéger le français non seulement dans les écoles et universités, mais dans toute la vie publique " (Parent, 1965, par. 621). Entre la création de la commission Parent en 1961 et la publication de son rapport de 1965, le gouvernement fédéral institua la Commission royale d'enquête sur le bilinguisme et le biculturalisme en 1963. Brièvement, celle-ci, aussi connue comme la commission Laurendeau-Dunton, recommanda en 1967 que le français et l'anglais soient officiels au Parlement, dans les cours fédérales et dans la fonction publique fédérale. L'année suivante, au Québec, la Commission scolaire de Saint-Léonard annonçait qu'elle cesserait d'offrir les classes bilingues et qu'elle les remplacerait progressivement par des classes en français, soulevant l'ire des parents allophones et anglophones. Le premier ministre Jean-Jacques Bertrand déposa un projet de loi garantissant le libre choix de la langue d'instruction en novembre 1968, lequel fut retiré quelques mois plus tard. Une violente manifestation 
de parents francophones et italiens conduisit à l'application de la Loi sur l'émeute en septembre 1969. Finalement, la Loi pour promouvoir la langue française au Québec fut adoptée en novembre 1969 (Robert, 2008, p. 306-307).

Ces événements constituent la crise de Saint-Léonard et ont contribué, à l'instar de la montée du nationalisme québécois, à instiguer l'action en matière linguistique de la part des deux ordres de gouvernement. Du côté du Canada, le gouvernement vota la Loi sur les langues officielles en juin 1969 (renforcée en 1985) qui établit le bilinguisme de la fonction publique fédérale, du Parlement, des cours fédérales (hormis la Cour suprême), des lois et fit des minorités francophones hors Québec et de la minorité anglophone au Québec la responsabilité du fédéral. Du côté du Québec, à la suite des recommandations de la Commission d'enquête sur la situation de la langue française et sur les droits linguistiques (commission Gendron), le gouvernement vota la Loi sur la langue officielle en 1974. Cette loi fit du français la langue officielle de la province, le rendit obligatoire dans l'affichage, força la francisation des entreprises, restreignit l'école anglaise aux enfants qui connaissaient déjà cette langue et accorda la priorité à la version française des lois québécoises. Puis, le Parti Québécois gagna les élections de 1976 et adopta la Charte de la langue française l'année suivante. Dans sa version initiale, cette loi prévoyait que le français serait la langue unique dans l'affichage, que les entreprises de cinquante employés et plus seraient francisées, que l'enseignement en anglais ne serait offert qu'aux enfants de parents ayant reçu leur enseignement primaire en anglais au Québec, que seule la version française des lois serait officielle. Ces dispositions ont été restreintes après des jugements et la Loi constitutionnelle de 1982. En matière d'affichage, dans le jugement Ford c. Québec de 1988, la Cour suprême statua que d'autres langues que le français devraient être permises. Le premier ministre Robert Bourassa usa de la disposition de dérogation de la Loi constitutionnelle de 1982 pour suspendre l'application de ce jugement. Pour l'enseignement, l'article 23 de cette même loi fit en sorte que les enfants puissent recevoir leur enseignement primaire et secondaire en anglais au Québec si leurs parents ou bien un frère ou une sœur avaient reçu leur instruction primaire (dans le cas des parents) ou encore primaire ou secondaire (dans le cas du frère ou de la sœur) en anglais au Canada. En 2009, la Loi modifiant la Charte de la langue française de 2002 (laquelle visait à restreindre l'enregistrement dans une école anglaise non subventionnée au Québec afin d'ensuite recevoir l'enseignement dans une école anglaise subventionnée par des fonds publics dans la province) fut invalidée par la Cour suprême qui jugea que « Le refus de prendre en compte le parcours d'un enfant dans une [école privée non subventionnée], imposé par l'al. 2 de l'art. 73 CLF [la Charte de la langue française de 1977], est total et sans nuance, et paraît excessif par rapport à la gravité du problème de l'accès quasi automatique aux écoles de la minorité linguistique par l'intermédiaire d'écoles passerelles " (Nguyen c. Québec, Cour suprême du Canada, 2009). Quant aux textes des lois québécoises, la Cour suprême jugea en 1979 dans la cause Québec c. Blaikie qu'il était inconstitutionnel que seule la version française des lois québécoises soit officielle (Cour suprême du Canada, 1979). En somme, la politique linguistique du Québec a été établie avec la Charte de la langue française de 1977. Depuis, des 
jugements et la Loi de 1982 ont diminué la prépondérance du français, mais cette langue demeure la seule langue officielle de la province.

La mise en œuvre des politiques linguistiques au Québec-Canada repose sur l'absence d'interaction entre les deux gouvernements. Lorsqu'interviewés (séparément), Guy Dumas, sous-ministre associé au Secrétariat à la politique linguistique du Québec de 1990 à 1995 et de 2000 à 2008, et Graham Fraser, commissaire au Commissariat aux langues officielles du Canada depuis 2006, ont été unanimes : il n'existe aucun contact ni entre les deux politiques, ni entre les autorités responsables de les mettre en œuvre depuis au moins les années 1990. Au cours de sa carrière, Dumas a participé à six changements de lois linguistiques et n'a jamais été témoin de contact avec le gouvernement fédéral ${ }^{3}$. Selon Fraser, le gouvernement fédéral ne veut pas avoir l'air de dire au Québec quoi faire en matière linguistique, car la question est trop sensible politiquement ${ }^{4}$.

\section{La politique d'immigration}

S'agissant de la politique d'immigration, la gouvernance multiniveau entre le Québec et le Canada fonctionne très bien. Depuis que le Québec a fait part de son intérêt pour l'immigration en créant le Service de l'immigration au sein du ministère des Affaires culturelles en 1965, le gouvernement fédéral a offert son appui à un partage des pouvoirs en matière d'immigration. En 1968, le secrétaire du Québec, Yves Gabias, et le ministre fédéral de la Citoyenneté et de l'Immigration, Jean Marchand, annoncèrent la création du nouveau ministère de l'Immigration du Québec. Officiellement, le fédéral collaborait parce qu'il reconnaissait la particularité linguistique du Québec et l'importance de préserver la langue française sur son territoire. Dans les faits, deux autres facteurs semblent avoir influencé la collaboration du gouvernement fédéral. D'abord, ce dernier devait sans doute savoir que, portée devant la justice, la demande du Québec pour bénéficier de plus de pouvoir en immigration serait reçue favorablement. En vertu du paragraphe 95 de la Loi constitutionnelle de 1867, l'immigration (de même que l'agriculture) est une compétence partagée entre les provinces et le fédéral. De plus, dans les années 1960, le mouvement nationaliste québécois gagnait du terrain et le fédéral avait intérêt à se soucier, du moins en apparence, du fait français au Québec. Ainsi, les discours des ministres québécois et canadien responsables de l'Immigration, lors de l'annonce du ministère québécois en 1968, révèlent un désir de collaboration entre les deux gouvernements (Radio-Canada, 1968).

La mise en œuvre de la gouvernance multiniveau au Québec-Canada a suivi un processus incrémental, c'est-à-dire qu'elle a avancé par changements marginaux. En 1968, lors de la première entente, la présence de fonctionnaires québécois dans les bureaux d'immigration canadiens à l'étranger fut accordée au gouvernement québécois afin qu'il puisse informer les immigrants de la réalité québécoise. En 1971, avec l'entente Cloutier-Lang, le gouvernement québécois put donner de l'information aux immigrants potentiels et acceptés que souhaitait admettre le gouvernement

\footnotetext{
3 Interview avec Guy Dumas en 2012.

4 Interview avec Graham Fraser en 2012.
} 
canadien sur le territoire québécois. L'entente Bienvenue-Andras de 1975 stipula que Québec et Ottawa planifieraient ensemble la politique d'immigration devant s'appliquer au Québec. De plus, les deux gouvernements partageraient l'information sur les demandes d'immigration et se consulteraient sur le recrutement et la sélection des résidents permanents et des travailleurs temporaires. Néanmoins, Ottawa prendrait la décision quant à la sélection des immigrants (Québec et Canada, 1975). En 1978, en vertu de l'entente Couture-Cullen, le gouvernement québécois put sélectionner ses immigrants dans certaines catégories : immigrants indépendants, travailleurs temporaires, étudiants, enseignants postsecondaires et touristes médicaux (Canada et Québec, 1978). À la suite de l'échec de l'Accord du lac Meech en 1990, le gouvernement fédéral souhaitait montrer qu'il faisait preuve de conciliation à l'égard du Québec et entreprit des négociations bilatérales avec le gouvernement québécois afin que ce dernier rapatrie des pouvoirs en matière d'immigration (Kelley et Trebilcock, 1998, p. 392-393). Depuis l'Accord Canada-Québec de 1991, la formule peut être résumée ainsi : sélection par le Québec et admission par le Canada. Plus précisément, le gouvernement québécois " sélectionne " les immigrants de toutes les catégories, sauf celles des réfugiés et de la réunification familiale, et le gouvernement canadien les " admet " en effectuant les vérifications médicales et de sécurité. Le Québec peut accepter autant d'immigrants que son poids démographique dans la population canadienne, plus $5 \%$. Finalement, le Québec reçoit un transfert fédéral pour l'intégration des immigrants, contrairement aux autres provinces où le fédéral est directement chargé de cette responsabilité (Canada et Québec, 1991). Malgré de nombreuses requêtes, aucune entrevue substantielle n'a pu être obtenue au ministère de l'Immigration et des Communautés culturelles du Québec. Par contre, une entrevue au ministère de la Citoyenneté et de l'Immigration du Canada a permis de confirmer que l'Accord Canada-Québec de 1991 guide l'ensemble des correspondances et des contacts entre les deux ministères. Les deux parties seraient totalement satisfaites de l'Accord et de la gouvernance qu'il permet, hormis certains éléments liés aux dates d'échéance qui ne conviendraient plus au calendrier actuel du gouvernement fédéral ${ }^{5}$. Autrement dit, mis à part quelques retards qui se répètent au fil des ans et contre lesquels le ministère de la Citoyenneté et de l'Immigration du Canada ne peut rien faire, la gouvernance multiniveau satisferait les deux ministères.

\section{La politique de relations internationales}

Les relations internationales font l'objet d'une gouvernance multiniveau partielle entre le Québec et le Canada. Dans certains dossiers, comme les organisations internationales, les deux paliers collaborent sur une base régulière et fonctionnelle, alors que dans d'autres, comme c'est le cas avec le commerce international, la collaboration est faible. Bien qu'elle soit partielle, cette gouvernance multiniveau est visible depuis le milieu des années 1980 et a été instaurée en partie grâce à l'intervention de la France (Bernier, 1996). Ainsi, en 1965, au cœur de

5 Interview avec un fonctionnaire anonyme du ministère de la Citoyenneté et de l'Immigration du Canada en 2012. 
la Révolution tranquille, le Québec signa avec la France deux accords sur des programmes d'échange en éducation et en culture, deux champs de compétence provinciale. La même année, le vice-premier ministre et ministre de l'Éducation du Québec, Paul Gérin-Lajoie, affirma dans un discours devant le corps consulaire à Montréal que le Québec avait juridiction sur ces compétences à l'international :

J'ai mentionné, il y a un instant, la surprise qu'a causée la signature, par la France et le Québec, d'une entente sur l'éducation. Cette entente est tout à fait conforme à l'ordre constitutionnel établi. Face au droit international, en effet, le gouvernement fédéral canadien se trouve dans une position unique. S'il possède le droit incontestable de traiter avec les puissances étrangères, la mise en œuvre des accords qu'il pourrait conclure sur des matières de juridiction provinciale échappe à sa compétence législative. Ainsi en a décidé, il y a près de trente ans [en 1937], un jugement du comité judiciaire du Conseil privé, jugement qui n'a jamais été infirmé. (Gérin-Lajoie, 1965)

La Loi constitutionnelle de 1867 stipule à l'article 132 que les relations internationales sont de compétence fédérale. Toutefois, le ministre se basa sur une décision du Comité judiciaire du Conseil privé de Londres, le plus haut tribunal canadien jusqu'en 1949 pour les questions de droit civil, remontant à 1937. Le tribunal avait alors statué, dans un jugement portant sur les relations de travail, que si un traité international s'intéressait à des compétences provinciales, le traité devait être mis en œuvre par les provinces. Autrement dit, le Canada ne peut s'engager dans un traité international sur des questions de compétence provinciale sans avoir obtenu l'aval des provinces (Russell, 1982, p. 122-123). C'est à cet élément de jurisprudence que fit référence Paul Gérin-Lajoie lorsqu'il prononça ce qui devint la " doctrine Gérin-Lajoie " : les compétences du Québec se " prolongent " à l'international. En 1968, Québec souhaitait prendre part à une conférence internationale sur l'éducation, un champ de compétence provinciale. La France organisa la présence du Québec en lui obtenant une invitation du pays hôte, le Gabon, à l'encontre de la volonté d'Ottawa. Québec prit part à la conférence en février et fut reçu avec les plus grands égards; or le Canada suspendit ses relations diplomatiques avec le Gabon et rappela son ambassadeur en poste dans le pays. En avril, le Québec participa à une autre conférence internationale, cette fois sur la francophonie, en France, et Ottawa fut informée une journée avant l'annonce publique par Québec (Sharp, 1968, p. 33-38). En 1969 et 1970, malgré la frustration du gouvernement canadien, le Québec participa à deux conférences internationales visant à créer l'Agence de coopération culturelle et technique, désormais l'Organisation internationale de la Francophonie. La France usa de son influence sur les pays africains et obtint une invitation formelle pour le Québec (Paquin, 2006). Finalement, en 1984, Brian Mulroney prit le pouvoir à Ottawa et signa, un an plus tard, un accord avec le nouveau premier ministre du Québec, Pierre-Marc Johnson. L'entente sur le Sommet francophone stipulait entre autres que :

Sur les questions relatives à la situation politique mondiale, le Premier ministre du Québec est présent et se comporte comme un observateur intéressé. Sur les questions relatives à la situation économique mondiale, le Premier ministre du Québec pourra, après concertation et avec l'accord ponctuel du Premier ministre du Canada, intervenir sur celles qui intéressent le Québec. (Canada et Québec, 1985) 
En d'autres termes, le Québec participerait à une conférence internationale et interviendrait si le sujet se rapportait à ses champs de compétence. Cette collaboration croissante fut stoppée en 1995, année du second référendum sur la souveraineté du Québec, avec la publication par Ottawa d'un document intitulé Le Canada dans le monde. En réponse à ce document, Québec publia en 1999 la Déclaration du gouvernement du Québec concernant la participation aux forums internationaux traitant d'éducation, de langue, de culture et d'identité, laquelle déplorait :

[...] dans son énoncé de 1995, Le Canada dans le monde, faisant de la culture un des trois piliers de son action internationale, le gouvernement du Canada ignore jusqu'à l'existence de la culture québécoise et que des ministres fédéraux la décrivent comme une simple " composante régionale " d'une culture canadienne unique [...] (Québec, 1999)

Puis, l'Assemblée nationale du Québec vota deux lois. D'abord, la Loi sur l'exercice des droits fondamentaux et des prérogatives du peuple québécois et de l'État du Québec de 2000 réitéra la doctrine Gérin-Lajoie :

L'État du Québec est libre de consentir à être lié par tout traité, convention ou entente internationale qui touche à sa compétence constitutionnelle.

Dans ses domaines de compétence, aucun traité, convention ou entente ne peut l'engager à moins qu'il n'ait formellement signifié son consentement à être lié par la voix de l'Assemblée nationale ou du gouvernement selon les dispositions de la loi.

Il peut également, dans ses domaines de compétence, établir et poursuivre des relations avec des États étrangers et des organisations internationales et assurer sa représentation à l'extérieur du Québec. (Québec, 2000, chap. II, par. 7)

Ensuite, la Loi modifiant la Loi sur le ministère des Relations internationales et d'autres dispositions législatives de 2002 précisa que tout accord international signé par le gouvernement fédéral et affectant les compétences du Québec devrait être approuvé par l'Assemblée nationale. Finalement, la bonne entente en matière de relations internationales fit un pas en avant en 2006, lorsque le premier ministre fédéral nouvellement élu, Stephen Harper, accorda au Québec de siéger à l'UNESCO. L'Accord Québec-Canada sur l'UNESCO prévoit un représentant permanent pour la province au sein de la délégation canadienne. En vertu de cette entente, le Québec a un " accès immédiat à tous les documents officiels transmis par l'UNESCO » et est donc en mesure d'influencer la position canadienne " en temps réel " (Québec, 2012).

La mise en œuvre de la gouvernance multiniveau en matière de relations internationales est caractérisée par sa nature différente selon les dossiers. Hormis ce qui concerne les organisations internationales, aucun accord formel entre Québec et Ottawa ne régit les contacts entre les deux gouvernements. Des entrevues réalisées avec des fonctionnaires québécois et fédéraux ont montré que pour ce qui est des organisations internationales, les fonctionnaires québécois et fédéraux collaborent, alors qu'ils ne le font pas pour le commerce international. En matière d'organisations internationales, Québec et Ottawa coopèrent à l'enseigne de plusieurs dossiers " comme s'ils travaillaient pour le même gouvernement ", au dire 
d'un fonctionnaire québécois ${ }^{6}$. L'information est partagée sans réserve et les dossiers seraient dorénavant mieux montés parce qu'ils sont étudiés de façon rigoureuse par deux groupes de fonctionnaires, c'est-à-dire de Québec et d'Ottawa ${ }^{7}$. Par contre, s'agissant de commerce international, la collaboration est irrégulière. Cela s'explique en partie par la Loi constitutionnelle de 1867, selon laquelle le fédéral est responsable du commerce international (art. 91, par. 2). Toutefois, les provinces sont chargées de l'application des accords internationaux lorsque ceux-ci touchent leurs champs de compétence; d'où la " clause Canada " que le gouvernement fédéral inclut dans les accords qu'il signe avec d'autres pays, le prémunissant contre une province qui refuserait d'appliquer un accord. Dans le cadre des négociations actuelles sur le libre-échange entre le Canada et l'Union européenne, les fonctionnaires québécois et fédéraux partagent l'information. De l'avis de fonctionnaires québécois et fédéraux interviewés, cette rare collaboration en matière de commerce international serait attribuable à la demande de l'Union européenne qui souhaite, dans le cadre de cet accord, accéder aux contrats publics des provinces, lesquels sont de compétence provinciale ${ }^{8}$. Pour les autres accords commerciaux, les provinces sont informées, mais pas consultées formellement. Un directeur général du gouvernement fédéral interviewé à ce sujet affirme que la plupart des pays, à l'exception de la France, de la Belgique et de la Suisse, ne connaissent pas le Québec et ne souhaitent négocier qu'avec le gouvernement fédéral ${ }^{9}$. Un fonctionnaire du gouvernement québécois a, quant à lui, indiqué que le gouvernement fédéral invite les provinces à s'exprimer sur le contenu des négociations commerciales ${ }^{10}$ (autres que celles avec l'Union européenne). En somme, il y a gouvernance multiniveau en matière de relations internationales entre Québec et Ottawa, mais celle-ci n'est pas formelle et son degré varie selon les dossiers.

\section{- LA GOUVERNANCE MULTINIVEAU EN CATALOGNE-ESPAGNE}

En Catalogne-Espagne, la gouvernance multiniveau était auparavant inexistante du côté des politiques linguistiques; la Catalogne devait respecter la co-officiliaté du castillan avec le catalan sur son territoire. Toutefois, des événements récents laissent croire que le gouvernement central tentera d'exercer plus de pouvoir en matière linguistique. Quant à l'immigration, la gouvernance multiniveau y est soumise, d'une part, à la gestion budgétaire de Madrid, qui transfère des fonds aux communautés autonomes chargées de l'accueil et de l'intégration des immigrants, et, d'autre part, au fonctionnement des entités responsables de la gouvernance multiniveau où siègent des élus politiques aux idées parfois différentes. Enfin, en ce qui concerne les relations extérieures, la gouvernance multiniveau accorde beaucoup de pouvoir à Madrid comparativement à Barcelone.

6 Interview avec un fonctionnaire anonyme du ministère des Relations internationales du Québec en 2012.

7 Interview avec un fonctionnaire anonyme du ministère des Relations internationales du Québec en 2012.

8 Interview avec Patrick Muzzi en 2012; interview avec Randle Wilson en 2012.

9 Interview avec Randle Wilson en 2012.

${ }^{10}$ Interview avec Patrick Muzzi en 2012. 


\section{La politique linguistique}

Officiellement, la Catalogne est responsable de sa politique linguistique, laquelle n'est donc pas soumise à la gouvernance multiniveau. La Constitution de 1978 de l'Espagne stipule à l'article 3 que tous les citoyens du pays ont le devoir de connaître le castillan et qu'il s'agit de la langue officielle du pays; autrement dit, Madrid veut pouvoir communiquer avec ses citoyens dans une seule langue. En revanche, le même texte stipule, dans le même article, que les communautés autonomes, telle la Catalogne, pourront choisir une autre langue comme étant coofficielle (avec le castillan) sur leur territoire. Dès 1979, la Catalogne s'est prévalue de ce droit : à l'article 3 de son Statut d'autonomie (lequel est, en quelque sorte, la Constitution de la communauté autonome), elle a déclaré que le catalan était la "langue propre " de son territoire et que le castillan et le catalan y seraient co-officiels. En 1983, la Catalogne vota la Loi sur la normalisation linguistique. Il revenait à la Generalitat (le gouvernement catalan) la responsabilité de promouvoir la «normalisation » du catalan, c'est-à-dire son égalité avec le castillan (Catalogne, 1983). À cette fin, la loi fit du catalan la langue de l'administration publique et du Parlement catalan et encouragea son utilisation dans les médias de communication (journaux, radio, livres, autres manifestations cultuelles). Plus important encore, la loi stipula que tous les enfants devaient être capables d'utiliser " normalement et correctement " les deux langues co-officielles à la fin de leur éducation primaire, mais qu'ils étaient libres de choisir l'école catalane ou castillane. Cette loi favorisant le catalan fut bien reçue en Catalogne et en Espagne. Les langues minoritaires (comme le catalan, le galicien et le basque) étaient interdites sous la dictature de Franco de 1939 à 1975; le regain de vitalité du catalan symbolisait la transition vers la démocratie de l'Espagne tout entière (Mar-Molinero, 2000, p. 160-161).

La législation linguistique suivante, soit le décret établissant l'aménagement de l'enseignement infantile, primaire et secondaire obligatoire en Catalogne de 1992, força tous les enfants à étudier en catalan à partir de l'âge de huit ans. Cette loi fut mal accueillie dans la société espagnole hors Catalogne. À la rentrée des classes en septembre 1993, le grand quotidien espagnol $A B C$ publia un article intitulé "Comme Franco, mais à l'envers : persécution du castillan en Catalogne " pour dénoncer ce qu'il percevait comme l'imposition du catalan aux personnes désireuses d'éduquer leurs enfants en castillan. En 1998, la Loi sur la politique linguistique visait à normaliser encore davantage l'utilisation du catalan dans l'administration publique, l'éducation, les médias, les industries culturelles ainsi que le monde socioéconomique (Catalogne, 1998, art. 1c). Les contrats et conventions collectives sont dorénavant rédigés dans la langue co-officielle choisie par les parties ou bien dans les deux langues en cas de désaccord. Les étudiants doivent démontrer leurs connaissances orales et écrites dans les deux langues co-officielles afin d'obtenir leur diplôme d'éducation secondaire. Des quotas linguistiques sont imposés pour la radio, la télévision et le cinéma et les entreprises doivent offrir des services dans les deux langues. En 2006, la Catalogne révisa son Statut d'autonomie; celui-ci contenait de nombreuses modifications dans les champs de politique linguistique, d'immigration et de relations extérieures. Le nouveau Statut fut voté à la Generalitat catalane avant de faire l'objet de négociations avec les membres du 
Parlement espagnol, les Cortès, ce qui conduisit à l'affaiblir aux yeux des nationalistes catalans. Néanmoins, le texte fut approuvé par $74 \%$ des votants catalans par référendum en 2006. L'article 6 prévoyait qu'en tant que « langue propre " des Catalans, le catalan « est la langue utilisée habituellement et de préférence par les administrations publiques et les médias publics de Catalogne. En outre, le catalan est normalement utilisé comme langue véhiculaire et d'apprentissage dans l'enseignement " (Catalogne, 2006a, art. 6.1). Selon la loi, l'Espagne devait prendre les moyens nécessaires pour faire reconnaître le catalan comme langue officielle dans l'Union européenne et pour que les citoyens catalans puissent communiquer avec le système judiciaire, avec les services du gouvernement central ainsi qu'avec les commerces situés en Catalogne dans leur langue. La même année, des membres du Parti populaire (PP, de droite) contestèrent la constitutionnalité de nombreux articles devant le Tribunal constitutionnel. Après moult tribulations, ce dernier rendit son jugement en 2010. Le nouveau Statut d'autonomie de la Catalogne s'est vu considérablement invalidé par ce jugement de dernière instance. La cour statua que le catalan ne pouvait être la " langue préférée " de la communauté autonome parce que cela diminuerait le statut du castillan. De plus, les commerces ne pouvaient être forcés de communiquer en catalan. En outre, le castillan devait être la langue normale d'enseignement au même titre que le catalan.

La mise en œuvre de la politique linguistique en Catalogne ne semble pas soumise à la gouvernance multiniveau, mais cette situation est peut-être en train de changer. La langue est officiellement du ressort de la Catalogne : la Generalitat est responsable de faire appliquer ses lois en matière de langue sur son territoire. Toutefois, la Catalogne doit s'assurer de respecter la co-officialité du castillan et du catalan prévue dans la Constitution et interprétée par le Tribunal constitutionnel. Selon le directeur général de la politique linguistique de la Generalitat de Catalogne de 1996 à 2003, lorsque la Loi sur la politique linguistique de 1998 a été préparée par la Generalitat, les interlocuteurs du gouvernement central se disaient inquiets au sujet de l'avenir du castillan dans la communauté autonome et craignaient pour son recul ${ }^{11}$. En 2010, le Tribunal constitutionnel a prévenu que le castillan ne devait pas occuper une place moindre que le catalan dans le secteur de l'éducation. En date de mars 2013, le ministre de l'Éducation espagnol (du Parti populaire), José Ignacio Wert, a déposé un projet de loi sur la qualité de l'éducation qui imposerait des évaluations régulières en castillan et un contenu commun correspondant à $65 \%$ des heures d'enseignement en Catalogne à partir de l'année scolaire 20142015. L'opposition est vive en Catalogne où le projet de loi est considéré comme favorisant un traitement inférieur du catalan et s'attaquant à une compétence de la communauté autonome.

\section{La politique d'immigration}

Bien qu'une gouvernance multiniveau semble exister en matière d'immigration en Catalogne-Espagne, elle ne fonctionne pas très bien en raison de sa gestion. Lorsque l'Espagne s'est dotée d'une Constitution en 1978, le pays faisait face à une

${ }^{11}$ Interview avec Lluís Jou en 2012. 
émigration plutôt qu'à une immigration. L'article 149.1 stipule que cette dernière est la compétence exclusive du gouvernement central. Au début des années 1990, l'Espagne est devenue un pays d'immigration nette et la Catalogne, communauté autonome riche, a commencé à recevoir de nombreux immigrants et à s'intéresser au phénomène sur son territoire. En 1993, cette dernière s'est dotée d'un comité interministériel sur l'immigration et d'un plan interministériel sur l'immigration 1993-2000, lequel mettait en place quelque quarante programmes destinés aux immigrants. En 2000, Madrid vota la Loi des droits et libertés des étrangers en Espagne et de leur intégration sociale qui faisait mention des communautés autonomes en deux endroits. D'abord, une Conférence sectorielle serait mise sur pied afin de coordonner les questions d'immigration avec les communautés autonomes. Ensuite, le ministre du Travail et de l'Immigration d'Espagne allait prendre en compte les projections de la main-d'œuvre demandée dans les communautés autonomes lorsqu'il déciderait du nombre de visas de travail annuels à délivrer, et la gestion des visas de travail se ferait dorénavant en collaboration avec les communautés autonomes. En 2000, la Generalitat se dota d'un Secrétariat à l'immigration et l'année suivante la Catalogne profita de la nouvelle loi pour établir ses compétences : elle publia un décret indiquant que son gouvernement était chargé des services sociaux (dont la santé, l'éducation et la formation professionnelle) offerts à tous les immigrants sur son territoire. Toujours en 2001, la Catalogne publia son nouveau Plan interministériel d'immigration 2001-2004 dans lequel elle rappelait sa compétence en matière de travail (en vertu de l'article 11.2 de son Statut d'autonomie de 1979) et affirmait ses compétences en immigration : "La Generalitat a une incidence qui doit être significative sur l'intégration sociale et économique de la population immigrante à travers les politiques d'éducation, de santé, de logement, etc. " (Catalogne, 2001 ${ }^{12}$ ). En 2006, forte de sa politique d'immigration mise en œuvre depuis 2000, la Catalogne inclut dans son nouveau Statut d'autonomie un article officialisant la constitutionnalité de ses interventions à l'égard des immigrants. L'article 138.1 stipule :

En matière d'immigration, la Generalitat a : a) une compétence exclusive, en matière de premier accueil des personnes immigrées, qui comprend les activités sociosanitaires et d'orientation; b) le développement de la politique d'intégration des personnes immigrées, dans le cadre de sa compétence; c) l'établissement et la réglementation des mesures nécessaires à l'intégration sociale et économique des personnes immigrées et à leur participation sociale; d) l'établissement par une loi d'un cadre de référence pour l'accueil et l'intégration des personnes immigrées. (Catalogne, 2006a)

Aussi, l'article 138.2 précise le pouvoir de la Catalogne quant aux permis de travail : "La Generalitat a une compétence exécutive en matière d'autorisation de travail aux étrangers dont la relation de travail s'établit en Catalogne " (Catalogne, 2006a). Pendant que le Tribunal constitutionnel étudiait le nouveau Statut d'autonomie, Barcelone tint une grande consultation publique sur l'immigration et en

12 Les traductions sont de l'auteure. 
tira un Pacte national pour l'immigration en 2008, démontrant son leadership et la gestion de ses compétences formellement acquises avec l'article 138. Le Plan de citoyenneté et d'immigration 2009-2012 mettant en œuvre le Pacte national compte plus de cent soixante pages et est disponible en français, en anglais et en aranais en plus du catalan et du castillan. Il formalise en quelque sorte la politique d'immigration de la Catalogne. Trois axes d'action sont identifiés : " Gestion des flux migratoires et accès au marché du travail ", " Adaptation des services publics à une société diverse " et "Intégration dans une culture publique commune " (Catalogne, 2009). En raison de la croissance de la législation européenne en immigration, ce plan prévoit à la fois davantage de responsabilité européenne et de dévolution vers les communautés autonomes en matière d'immigration :

[L]a prise en charge par l'Union européenne d'importantes compétences en matière d'immigration et d'asile [...] déplace progressivement les compétences des États membres [telle l'Espagne], en particulier dans les fonctions d'élaboration de politiques communautaires, l'adoption de législation et planification et, plus exceptionnellement, d'exécution. Et, dans les États décentralisés, également des niveaux de gouvernement sous-nationaux, comme ceux des communautés autonomes. (Catalogne, 2009, p. 44)

Effectivement, en 2009, l'Espagne vota une nouvelle loi des droits et libertés des étrangers en Espagne et de leur intégration sociale (cette loi remplace dorénavant celle du même nom datant de 2000). L'une des raisons de cette refonte tenait aux neuf directives européennes liées à l'immigration prescrites depuis la dernière loi; l'Espagne, comme les autres États membres de l'Union européenne, devait les inclure dans sa législation. La loi créa du même coup une Conférence sectorielle en immigration afin de " canaliser la coopération entre l'Administration générale de l'État [espagnol] et les communautés autonomes " (Espagne, 2009, art. VII). Elle reconnut aussi le rôle des communautés autonomes pour ce qui est de l'intégration des immigrants. Or, en 2010, le Tribunal constitutionnel restreint l'interprétation de l'article 138 (portant sur l'immigration) du Statut d'autonomie de 2006. La cour avisa que la Catalogne pouvait s'impliquer en immigration (en offrant des services sociaux aux immigrants), mais qu'elle ne pouvait choisir les travailleurs immigrants (elle peut appliquer les règles dictées par Madrid). De plus, le Tribunal a indiqué qu'il revenait au gouvernement central de fixer les termes de la participation de la Catalogne aux décisions en matière d'immigration. En somme, avec la sentence de 2010, la Catalogne a vu ses espoirs diminuer quant à une gouvernance multiniveau plus formelle en immigration.

La mise en œuvre de la gouvernance multiniveau de la politique d'immigration repose sur la volonté de collaboration de Madrid, qui détient l'ensemble des pouvoirs dans ce domaine et distribue aussi les ressources financières. Depuis 2009, la Catalogne peut délivrer les autorisations de travail aux immigrants en suivant les règles dictées par Madrid. La Conférence sectorielle en immigration, où les communautés autonomes et le gouvernement central discutent d'immigration, s'est réunie six fois depuis sa création (Espagne, 2012b). Le rapport de la dernière 
rencontre disponible tenue en $2011^{13}$ fait état de deux points d'information transmis par le gouvernement central aux communautés autonomes : présentation de modifications apportées à un règlement et approbation du Fonds d'appui à l'accueil et à l'intégration des immigrants (Espagne, s.d., p. 38). Enfin, la Commission bilatérale Catalogne-Espagne, qui existe depuis 2006, comprend une sous-commission bilatérale en matière d'immigration. Celle-ci " analyse les questions de travail et de résidence des étrangers qui touchent directement la Catalogne " (Espagne, s.d.). L'information sur le nombre de réunions de cette sous-commission n'est pas disponible. De plus, parce que les membres sont des élus politiques, la coopération est moindre en cas de divergence entre les partis politiques. Quant aux ressources financières, la compétence constitutionnelle de la Catalogne pour l'accueil et l'intégration des immigrants est circonscrite par le financement provenant du gouvernement central. En 2012, les ressources financières devant permettre aux communautés autonomes de mener à bien ces tâches, le Fonds d'appui à l'accueil et à l'intégration des immigrants, ont été annulées (EFE, 2012). La gouvernance multiniveau en matière d'immigration existe-t-elle ? Puisque la Catalogne reçoit l'argent de la part de Madrid, laquelle peut l'annuler sans consultation, et puisque le gouvernement central n'est pas tenu de consulter les communautés autonomes sur les questions d'immigration (selon le jugement de 2010), Barcelone se trouve dans une position qui s'apparente plutôt à une gouvernance " espagnole " que multiniveau.

\section{La politique de relations extérieures}

Selon l'article 149.1.3 de la Constitution espagnole, les relations internationales sont du ressort du gouvernement central. Néanmoins, depuis la fin des années 1990, la Catalogne est active dans ce domaine. Elle a obtenu davantage de pouvoir en matière de relations internationales grâce à l'interprétation de la Constitution par le Tribunal constitutionnel au cours des années 1990. Auparavant, en 1985, ce dernier avait statué que la Galice (une autre communauté autonome) avait outrepassé ses compétences en signant un communiqué de collaboration avec le Danemark, un pays. Toutefois, le juge Eugenio Díaz Eimil émit un jugement divergent dans lequel il argua que la Galice devrait pouvoir poursuivre ses activités internationales si celles-ci relevaient de ses compétences et n'affectaient pas la souveraineté espagnole (Tribunal Constitucional, 1989a, Voto). Ce jugement porta ses fruits. La même année, la Catalogne contesta un décret espagnol selon lequel seule Madrid pouvait déterminer un film " espagnol " en coproduction. Barcelone défendit que les arts relevaient de son champ de compétence et qu'elle était responsable de mettre en œuvre les traités internationaux affectant ses compétences selon l'article 27.3 de son Statut d'autonomie de 1979. Le Tribunal constitutionnel reconnut que la Catalogne pouvait établir la nationalité d'un film. Plus intéressant encore, la cour statua, à l'instar du juge Díaz Eimil quelques mois plus tôt, que les " relations extérieures » ne constituaient pas toutes des " relations internationales "

${ }^{13}$ Une réunion de la Conférence sectorielle d'immigration s'est tenue le 27 juin 2012, mais le rapport n'est pas accessible (Espagne, 2012a). 
et n'étaient donc pas toutes assujetties à l'article 149.1.3 de la Constitution (Tribunal Constitucional, 1989b).

Cinq années plus tard, en 1994, le même tribunal jugea que le Pays basque (une autre communauté autonome) pouvait maintenir son bureau de lobbying destiné aux membres de l'Union européenne à Bruxelles. Cette fois-ci, le Tribunal constitutionnel détailla son interprétation des " relations internationales " et jugea que le bureau basque relevait des " activités extérieures ", donc était acceptable en vertu de la Constitution. La Catalogne, dont le bureau à Bruxelles était alors une " fondation ", devint une délégation avec le mandat officiel de lobbying auprès de l'Union européenne en 2004 (Catalogne, s.d.). En 2006, à l'article 183 de son nouveau Statut d'autonomie, une commission bilatérale entre Barcelone et Madrid fut prévue afin d'assurer, entre autres sur les questions de relations extérieures : "a) la participation et la collaboration de la Generalitat à l'exercice des compétences étatiques qui concernent l'autonomie de la Catalogne; b) l'échange d'information et l'établissement, le cas échéant, de mécanismes de collaboration aux politiques publiques respectives et aux questions d'intérêt commun " (Catalogne, 2006a). En 2010, le Tribunal constitutionnel affirma que : " la participation [de la Generalitat à la commission bilatérale devra] être régie par la législation du gouvernement central et ne pourra se substituer aux organes décisionnels de l'État [central] ni porter atteinte au libre et plein exercice des compétences du gouvernement central " (Tribunal Constitucional, 2010 par. 111). En d'autres termes, la Catalogne devra se soumettre aux organes décisionnels du gouvernement espagnol, et ce dernier ne sera pas tenu de consulter la Catalogne si cela affecte ses propres compétences.

La gouvernance de la mise en œuvre de la politique de relations extérieures est formelle, mais laisse peu de pouvoir à la Catalogne en regard de celui accordé à l'Espagne. Le Traité sur l'Union européenne de 1992 encouragea la participation des régions aux négociations des politiques européennes. L'Espagne signa donc en 1992 l'Accord institutionnalisant la conférence sur les affaires relatives aux communautés européennes. Cette conférence, la CARCE, a pour objectif de " s'attaquer, en respectant le principe de la coopération [entre les communautés autonomes et le gouvernement central], à résoudre progressivement les questions de la participation des communautés autonomes à l'élaboration et à l'application du droit et des politiques européennes " (Espagne, 1992). Autrement dit, la CARCE est le lieu de rencontre sur la mise en œuvre de normes européennes. L'Accord fut révisé en 1994 et en 1997 et, depuis, prévoit au moins deux rencontres par an (Espagne, 1997). De plus, depuis 2004 deux " conseillers » nommés par la CARCE informent les communautés autonomes des dossiers pertinents et font valoir leur position commune en siégeant à la Représentation permanente de l'Espagne devant l'Union européenne. Aussi depuis 2004, la CARCE prend part aux discussions de quatre (cinq depuis 2009) groupes de travail du Conseil de l'Union européenne (Espagne, 2005 et 2010). Selon la secrétaire aux Affaires extérieures de la Catalogne, lorsque toutes les communautés autonomes sont d'accord, leur position est forte auprès du gouvernement central. Néanmoins, la CARUE (nommée ainsi depuis 2010) s'intéresse surtout à des questions mineures et une position commune est parfois difficile à atteindre étant donné le caractère politique de ses membres. 
Les représentants sont des élus des communautés autonomes et du gouvernement central, donc les élus des communautés autonomes du même parti que ceux du gouvernement central ont tendance à s'entendre sur les questions épineuses ${ }^{14}$. Il n'est pas sans intérêt de mentionner qu'aux dernières élections espagnoles de 2011, seules quelques communautés autonomes - dont la Catalogne - ont voté pour un parti autre que le Parti populaire, marginalisant leur position à la CARUE. Enfin, la Commission bilatérale Catalogne-Espagne prévue dans le Statut d'autonomie de 2006 a créé la sous-commission sur l'Union européenne, laquelle serait par contre peu active depuis l'arrivée au pouvoir du Parti populaire en $2011^{15}$.

\section{- CONCLUSION}

À la lumière de notre recherche, il ressort que la gouvernance multiniveau au Québec-Canada et en Catalogne-Espagne est à son sommet avec la politique d'immigration du premier cas. L'Accord Canada-Québec de 1991 établit formellement et précisément les modalités de la politique; il guide l'ensemble des relations entre les deux paliers de gouvernement. À l'opposé, la gouvernance multiniveau semble désordonnée et peu efficace en Catalogne-Espagne. Les échanges entre les deux ordres de gouvernement sont placés entre les mains d'élus qui, dès lors qu'ils sont d'allégeance différente, s'avèrent susceptibles d'éprouver une certaine difficulté à s'entendre.

La conjoncture entourant les relations entre la Catalogne et l'Espagne ne facilite pas la gouvernance multiniveau. Depuis le rejet en 2010 de nombreux articles du Statut d'autonomie de 2006 par le Tribunal constitutionnel, la Catalogne est mécontente du gouvernement central, de surcroît dirigé depuis 2011 par le Parti populaire, lequel apporta devant le Tribunal le Statut révisé. La crise financière que traverse l'Espagne ajoute à la tension; la Catalogne, communauté autonome riche, revendique le droit de conserver une plus grande part des recettes fiscales perçues sur son territoire. En somme, plus rien ne va entre les gouvernements de Barcelone et de Madrid : cette tension se reflète certainement dans la gouvernance multiniveau qui semble bien mal fonctionner selon l'analyse présentée. Sans cette tension, la gouvernance multiniveau serait probablement - ou du moins en apparence - plus fonctionnelle.

Au final, les relations entre une nation minoritaire et le gouvernement fédéral sont sujettes, pour l'ensemble des champs de politiques publiques, aux événements politiques et économiques. Nous avons défini la gouvernance multiniveau comme l'ensemble des processus par lesquels les deux ordres de gouvernement agencent leur politique respective dans un champ de politique donné. Lorsqu'elle repose sur des documents officiels et acceptés par les deux parties, elle permet de restreindre les contrecoups d'événements politiques et économiques sur les relations entre les deux gouvernements. En guise d'illustration, la politique de relations internationales du Québec quant aux organisations internationales est régie par un accord formel, lequel offre un faible risque de modification, peu importe les

\footnotetext{
${ }^{14}$ Interview avec Débora Miralles Solé en 2012.

15 Interview avec Débora Miralles Solé en 2012.
} 
événements politiques et économiques. La gouvernance multiniveau entre Québec et Ottawa ne peut qu'y gagner, c'est-à-dire qu'elle peut être plus efficace et moins conflictuelle. Au contraire, entre Barcelone et Madrid, tous les champs de politique étudiés affichent une gouvernance défaillante en raison des événements politiques et économiques actuels qui ne sont pas compensés par une gouvernance multiniveau formelle.

\section{BIBLIOGRAPHIE}

ABC (1993). « Igual que Franco, pero al revés: persecución del castellano en Cataluña », $A B C$, p. 1.

Bernier, L. (1996). De Paris à Washington. La politique internationale du Québec, Sainte-Foy, Presses de l'Université du Québec.

Cour suprême du Canada (2009). Nguyen c. Québec (Éducation, Loisir et Sport), www. jugements.qc.ca/php/decision.php ?liste=65866289\&doc=79ABE1656F137663D2C B25368D9DFD8B8366457024FC6B2797F6FF8803D9F6FA\&page=1 (page consultée le 7 janvier 2013).

Cour suprême du Canada (1979). Procureur général du Québec c. Blaikie et autres, http://scc. lexum.org/decisia-scc-csc/scc-csc/scc-csc/fr/item/2637/index.do (page consultée le 14 mars 2013).

Canada (1982). Loi constitutionnelle de 1982, http://laws-lois.justice.gc.ca/fra/Const/ (page consultée le 13 mars 2013).

Canada (1867). Loi constitutionnelle de 1867, http://laws-lois.justice.gc.ca/fra/Const/ (page consultée le 13 mars 2013).

Canada et Québec (1991). Accord Canada-Québec relatif à l'immigration et à l'admission temporaire des aubains, www.micc.gouv.qc.ca/publications/pdf/Accord_canada_ quebec_immigration_francais.pdf (page consultée le 12 mars 2013).

Canada et Québec (2006). Accord entre le gouvernement du Canada et le gouvernement $d u$ Québec relatif à l'Organisation des Nations Unies pour l'éducation, la science et la culture (UNESCO), http://pm.gc.ca/fra/media.asp ?id=1153 (page consultée le 14 mars 2013).

Canada et Québec (1985). Entente entre le gouvernement du Québec et le gouvernement du Canada relative au Sommet francophone, www.saic.gouv.qc.ca/publications/Positions/ Partie3/Document21.pdf (page consultée le 28 juin 2011).

Canada et Québec (1978). Entente entre les gouvernements du Canada et du Québec portant sur la collaboration en matière d'immigration et sur la sélection des ressortissants étrangers qui souhaitent s'établir au Québec à titre permanent ou temporaire.

Canada et Québec (1975). Entente portant sur l'échange de renseignements, le recrutement et la sélection des ressortissants étrangers qui demeurent à l'extérieur du Canada et qui désirent résider de façon permanente dans la province de Québec ou être admis à titre temporaire pour y exercer un emploi.

Canada et Québec (1971). Entente portant sur la présence d'agents d'orientation du ministère de l'Immigration du Québec dans les bureaux fédéraux d'immigration à l'extérieur du Canada. 
Catalogne (s.d.). Brusselles, Secretaria de Relacions Internacionals, www.gencat.cat/ relacions_exteriors/oficines/6_3_bruseles.htm (page consultée le 7 janvier 2013).

Catalogne (2009). Plan de citoyenneté et d'immigration 2009-2012, www20.gencat.cat/docs/ dasc/03Ambits\%20tematics/05Immigracio/03Politiquesplansactuacio/03placiuta dania09_012/01Presentacio/PCIFRANCES.pdf (page consultée le 7 janvier 2012).

Catalogne (2006a). Loi organique no 6/2006, du 19 juillet, de réforme du Statut d'autonomie de Catalogne, www.parlament.cat/porteso/estatut/estatut_frances_100506.pdf (page consultée le 14 janvier 2013).

Catalogne (2006b). Estatut d'autonomia de 2006, http://www.gencat.cat/generalitat/cat/ estatut/index_2006.htm (page consultée le 13 mars 2013).

Catalogne (2001). Pla Interdepartamental d'Immigració 2001-2004, www20.gencat.cat/docs/ dasc/03Ambits\%20tematics/05Immigracio/03Politiquesplansactuacio/01antecede nts/Enllasos/IMMIGR1.pdf (page consultée le 9 novembre 2011).

Catalogne (1998). Ley 1/1998, de 7 de enero, de política lingüística, http://www20.gencat. cat/portal/site/Llengcat/menuitem.1ab5a94fef60a1e7a129d410b0c0ela0/ ?vgne xtoid=4d67f9465ff61110VgnVCM1000000b0cle0aRCRD\&newLang=es_ES （page consultée le 12 mars 2013).

Catalogne (1992). Decret 75/1992, de 9 de març, pel qual s'estableix l'ordenació general dels ensenyaments de l'educació infantil, l'educació primària i l'educació secundària obligatòria a Catalunya, http://educacio.gencat.net/extranet/dogc/decret_75_1992.html (page consultée le 14 mars 2013).

Catalogne (1983). Llei 7/1983, de 18 d'abril, de normalització lingüística a Catalunya, http:// www20.gencat.cat/portal/site/Llengcat/menuitem.b318de7236aed0e7a129d410b 0c0e1a0/ ?vgnextoid=12d7f9465ff61110VgnVCM1000000b0c1e0aRCRD\&vgnextch annel=12d7f9465ff61110VgnVCM1000000b0cle0aRCRD\&vgnextfmt=default (page consultée le 12 mars 2013).

Catalogne (1979). Estatut d'autonomia de 1979, http://www.gencat.cat/generalitat/cat/ estatut1979/index.htm (page consultée le 13 mars 2013).

EFE (2012). " La Generalitat denuncia que no tendrá presupuesto para políticas de acogida ", La Vanguardia, www.lavanguardia.com/politica/20120504/54288703987/ generalitat-denuncia-no-tendra-presupuesto-politicas-acogida.html (page consultée le 19 juin 2012).

Espagne (s.d.). Cataluña: Secretaría de Estado de Administraciones Públicas, Ministerio de Hacienda y Administraciones Públicas, www.seap.minhap.gob.es/es/areas/ politica_autonomica/coop_bilateral_CCAA/comisiones_bilaterales/Catalunya. html (page consultée le 7 janvier 2013).

Espagne (s.d.). Informe sobre la actividad de las Conferencias sectoriales durante 2011, Madrid, Ministerio de Hacienda y Administraciones Públicas, www.seap.minhap.gob.es/ dms/es/areas/politica_autonomica/coop_multilateral_ccaa_ue/cooperacion_ multilateral/Conf_Sectoriales/Documentacion/Conf_Sect_anuales/parrafo/0/ text_es_files/INFORME-CONFERENCIAS-SECTORIALES-2011/INFORME\%20 CONFERENCIAS\%20SECTORIALES\%202011.pdf (page consultée le 7 janvier 2013). 
Espagne (2012a). Conferencias sectoriales - Reuniones en el año 2012, www.seap.minhap.gob. es/dms/es/areas/politica_autonomica/coop_multilateral_ccaa_ue/cooperacion_ multilateral/Conf_Sectoriales/Documentacion/Conf_Sect_Reuniones/parrafo/12/ conferencias_sectoriales_2012.pdf (page consultée le 7 janvier 2013).

Espagne (2012b). Conferencias sectoriales. Totales 1981/2012, www.seap.minhap.gob.es/ dms/es/areas/politica_autonomica/coop_multilateral_ccaa_ue/cooperacion_ multilateral/Conf_Sectoriales/Documentacion/Conf_Sect_Reuniones/ parrafo/81-12/conferencias_sectoriales_1981-2012.pdf (page consultée le 7 janvier 2013).

Espagne (2010). Conferencia para Asuntos Relacionados con la Unión Europea, Ministerio de Política Territorial y Administración Pública, www.mpt.gob.es/areas/politica_ autonomica/coop_multilateral_ccaa_ue/ccaa_y_ue/Participacion_CCAA_ Consejo_Ministros/CARUE (page consultée le 11 mai 2011).

Espagne (2009). Ley Orgánica 2/2009, de 11 de diciembre, de reforma de la Ley Orgánica 4/2000, de 11 de enero, sobre derechos y libertades de los extranjeros en España y su integración social, www.boe.es/boe/dias/2009/12/12/pdfs/BOE-A-2009-19949.pdf (page consultée le 14 janvier 2013).

Espagne (2005). Resolución de 28 de febrero de 2005, de la Secretaría de Estado de Cooperación Territorial, por la que se ordena la publicación de los Acuerdos de 9 de diciembre de 2004, de la Conferencia para Asuntos Relacionados con las Comunidades Europeas, sobre la Consejería para Asuntos Autonómicos en la Representación Permanente de España ante la Unión Europea y sobre la participación de las Comunidades Autónomas en los grupos de trabajo del Consejo de la Unión Europea; y sobre el sistema de representación autonómica en las formaciones del Consejo de la Unión Europea, www.boe.es/diario_boe/txt.php ?id=BOE-A-2005-4388 (page consultée le 12 mars 2013).

Espagne (2000). Ley Orgánica 4/2000, de 11 de enero, sobre derechos y libertades de los extranjeros en España y su integración social, http://noticias.juridicas.com/base_datos/ Admin/lo4-2000.html (page consultée le 14 janvier 2013).

Espagne (1997). Ley 2/1997, de 13 de marzo, por la que se regula la Conferencia de Asuntos Relacionados con las Comunidades Europeas, www.mpt.gob.es/areas/politica_ autonomica/coop_multilateral_ccaa_ue/ccaa_y_ue/Participacion_CCAA_ Consejo_Ministros/CARUE/ley_2_1997/document_es/documento.pdf (page consultée le 11 mai 2011).

Espagne (1992). Acuerdo de institucionalización de la Conferencia para Asuntos Relacionados con las Comunidades Europeas, www.cepc.es/rap/Publicaciones/Revistas/5/RIE_020_ 001_445.pdf (page consultée le 10 mai 2011).

Espagne (1978). Constitución española, www.congreso.es/consti/constitucion/indice/ index.htm (page consultée le 13 mars 2013).

Gérin-Lajoie, P. (1965). Allocution du ministre de l'Éducation, M. Paul Gérin-Lajoie, aux membres du Corps consulaire de Montréal, lundi, le 12 avril à 12 h 30 de l'après-midi, à l'hôtel Windsor, www.mrifce.gouv.qc.ca/Content/documents/fr/discours_gerin_lajoie.pdf (page consultée le 28 mars 2011).

Kelley, N. et M. Trebilcock (1998). The Making of the Mosaic: A History of Canadian Immigration Policy, Toronto, University of Toronto Press. 
Mar-Molinero, C. (2000). The Politics of Language in the Spanish-speaking World, London, Routledge.

Paquin, S. (2006). " La relation Québec-Paris-Ottawa et la création de l'Organisation de la Francophonie (1960-2005) ", Guerres mondiales et conflits contemporains, vol. 3, $\mathrm{n}^{\circ} 223$, p. 31-47.

Parent, A.-M. (1965). Rapport de la Commission royale d'enquête sur l'enseignement dans la province de Québec, t. 2, vol. 3, chap. XII, Québec, Publications du Québec.

Québec (2012). Accord Québec-Canada sur l'UNESCO, www.mrifce.gouv.qc.ca/fr/relationsdu-quebec/organisations-et-forums/representation-unesco/accord-unesco (page consultée le 6 janvier 2013).

Québec (2000). Loi sur l'exercice des droits fondamentaux et des prérogatives du peuple québécois et de l'État du Québec, www2.publicationsduquebec.gouv.qc.ca/dynamicSearch/ telecharge.php ?type=5\&file=2000C46F.PDF (page consultée le 12 mars 2013).

Québec (1999). Déclaration du gouvernement du Québec concernant la participation du Québec aux forums internationaux traitant d'éducation, de langue, de culture et d'identité, www. saic.gouv.qc.ca/publications/Positions/Partie3/Document39.pdf (page consultée le 28 juin 2011).

Radio-Canada (1968). Le Québec se dote d'un ministère de l'Immigration, http://archives. radio-canada.ca/politique/provincial_territorial/clips/12445/ (page consultée le 27 avril 2012).

Robert, J.-C. (2008). " La langue, enjeu politique du Québec ", dans M. Plourde et P. Georgeault (dir.), Le français au Québec : 400 ans d'histoire et de vie, Montréal, Fides, p. 301-309.

Russell, P. H. (1982). Leading Constitutional Decisions: Cases on the British North America Act, Ottawa, Carleton University Press.

Sharp, M. (1968). Federalism and International Conferences on Education: A Supplement to Federalism and International Relations, Ottawa, Queen's Printer and Controller of Stationery.

Tribunal Constitucional (2010). Sentencia 31/2010, de 28 de junio de 2010, www. tribunalconstitucional.es/fr/jurisprudencia/Pages/Sentencia.aspx ?cod=9873 (page consultée le 14 janvier 2013).

Tribunal Constitucional (1989a). Sentencia 137/1989, www.tribunalconstitucional.es/es/ jurisprudencia/Paginas/Sentencia.aspx ?cod=17969 (page consultée le 14 janvier 2013).

Tribunal Constitucional (1989b). Sentencia 153/1989, www.tribunalconstitucional.es/es/ jurisprudencia/Paginas/Sentencia.aspx ?cod=17985 (page consultée le 14 janvier 2013). 